\title{
Empowerment als Schlüssel für die agile Arbeitswelt
}

\section{Ansatzpunkte für ein konsequentes Mitarbeitenden-Empowe rment}

\author{
Andreas Boes, Katrin Gül, Tobias Kämpf und Thomas Lühr
}

\begin{abstract}
Zusammenfassung
Mit der digitalen Transformation stehen zahlreiche Unternehmen vor der Herausforderung, Neuland gestalten zu lernen. Richtungsweisend wirkt dabei das Leitbild der ,,agilen Organisation“" mit einer ausgeprägten Kundenorientierung, beschleunigten Innovationszyklen und enger Kollaboration. In diesem Umbruch entstehen neue Anforderungen an die Beschäftigten: Die Mitarbeitenden sollen fachübergreifend zusammenarbeiten, selbstorganisiert agieren und eine hohe Veränderungsbereitschaft mitbringen. Diese Phase der Neueinstellung bietet die Chance, ein konsequentes Empowerment der Mitarbeitenden zu fördern. Dafür muss allerdings der Autonomiezuwachs auf Seiten der Beschäftigten in Organisationsstrukturen eingebettet sein, die echte Handlungsfähigkeit sowie nachhaltige Arbeitsbedingungen ermöglichen. In dem Beitrag werden Ansatzpunkte für eine Stärkung des Empowerments von Beschäftigten vorgestellt.
\end{abstract}

\subsection{Die digitale Transformation: Unternehmen erfinden sich neu}

Die digitale Transformation markiert einen grundlegenden Umbruch für die Organisation von Arbeit - historisch vergleichbar mit der industriellen Revolution im 19. Jahrhundert. Die Auswirkungen dieses Umbruchs betreffen jedoch nicht vor allem die unmittelbare Fertigung (vgl. z. B. Hirsch-Kreinsen 2014), wie durch die Zuspitzung der Diskussion auf „Industrie 4.0“ oft impliziert wird, sondern sie reichen tief in die sogenannten ,indirekten“

A. Boes · K. Gül $(\bowtie) \cdot$ T. Kämpf · T. Lühr

ISF München e.V., München, Deutschland

E-Mail: andreas.boes@isf-muenchen.de; katrin.guel@isf-muenchen.de;

tobias.kaempf@isf-muenchen.de; thomas.luehr@isf-muenchen.de 
Kopfarbeitsbereiche der Angestellten hinein. Die Veränderungen gehen weit über die bloße Frage der Automatisierung und des Verlusts von Arbeitsplätzen (vgl. dazu Frey und Osborne 2013; Brynjolfsson und McAfee 2011) hinaus. Sie stellen vielmehr insgesamt die bisherige Organisation von Arbeit und die Gestaltung von Innovationsprozessen bis hin zur Steuerung von Wertschöpfung infrage (vgl. für einen guten Überblick: BMAS 2015).

Im Zuge dieses Umbruchs sind die Unternehmen gegenwärtig dabei, sich neu zu erfinden. Sie suchen nach einem Bauplan für die digitale Transformation. Als Leitbild kristallisiert sich die ,agile Organisation“ (Boes et al. 2016) heraus, die einen Gegenentwurf zum fordistisch-bürokratischen Unternehmen mit seinen hierarchischen Entscheidungsprozessen und abgeschotteten „Silos“ darstellt und eine Antwort auf die zunehmende Komplexität und Geschwindigkeit in den Unternehmensprozessen gibt. Den Hintergrund dieser Entwicklung bildet ein Produktivkraftsprung: So ist mit dem Aufstieg des Internets ein digitaler „Informationsraum“ (Baukrowitz und Boes 1996) entstanden, der die abstrakte Welt der Daten und Informationen mit der Lebendigkeit einer neuen gesellschaftlichen Handlungsebene verbindet (vgl. Boes 1996). Als Fundament für die Arbeits- und Produktionsprozesse im 21. Jahrhundert kommt ihm dieselbe Bedeutung zu wie den Maschinensystemen im 19. und 20. Jahrhundert. Mit ihm wird die Informationsebene zum strategischen Zentrum für die Steuerung der Geschäfts- und Produktionsprozesse, die von der manuellen Fertigung bis zur Kopfarbeit in den Büros - entlang des ,flow of information“ neu organisiert werden (vgl. Boes und Kämpf 2012). Als neuer „Raum der Produktion“ (Boes 2004) bildet er zudem die Grundlage für neue Formen der Kollaboration, insbesondere in den indirekten Bereichen.

In dieser Phase der Neufindung experimentieren die Unternehmen mit vielfältigen agilen Konzepten und Methoden. Beispiele hierfür sind etwa die Einführung agiler Methoden, wie Scrum oder Kanban (siehe Infokasten), die Nutzung Community-basierter Ansätze von Wissenstransfer oder verschiedenste Formen eines agilen „Staffings“, wie etwa Crowd Working bzw. die Nutzung von Formen interner „Crowd Work“ (vgl. Simmert et al. in diesem Band; Durward et al. 2019) bis hin zu Formen der Schwarmorganisation. Gemeinsam ist diesen unterschiedlichen Ansätzen vor allem eines: Sie sind Ausdruck einer Suche nach Alternativen zu bürokratischen Organisationskonzepten. Es geht dabei vor allem um das Aufbrechen starrer Abteilungsgrenzen, um „flache Hierarchien“, um mehr Flexibilität und um eine stärkere Kundenorientierung.

Auf der Ebene der Arbeitsorganisation kommt dabei der Übertragung von ,,agilen Methoden“" aus der Software-Entwicklung eine strategische Bedeutung zu. Agile Methoden haben sich mittlerweile in breitem Maßstab in der IT-Welt durchgesetzt. Sie kommen auch in den großen Unternehmen wie Google, IBM, Microsoft oder SAP in der Fläche zum Einsatz (vgl. z. B. Woodward et al. 2010; Sutherland und Schwaber 2011; Dingsøyr et al. 2010) - vor allem in Gestalt von „Scrum“, das als eine Art „Grassroot“- Bewegung gegen die Bürokratisierung der Software-Entwicklung entstanden war. 


\section{Was sind agile Methoden?}

Neue Formen agiler Software-Entwicklung wurden seit Mitte der 1990er-Jahre von Pionieren wie Ken Schwaber, Mike Beedle oder Jeff Sutherland konzipiert und in Projekten angewendet (vgl. z. B. Beedle und Schwaber 2002; Sutherland und Schwaber 2011). Neben dem „Pair Programming“", das auf eine Verbesserung des Wissensaustauschs im Arbeitsprozess zielt, und „Test Driven Development" zur frühzeitigen Fehleridentifizierung hat sich in der Praxis vor allem Scrum als agile Methode herausgebildet. Als agile Methode der Projektorganisation stellt Scrum einen Gegenentwurf zu den lange Zeit vorherrschenden, bürokratischen Wasserfallprojekten mit ihren langen Planungs- und teils mehrjährigen Projektlaufzeiten dar (zur Kritik vgl. exemplarisch DeMarco und Lister 1987). Es basiert auf der Grundidee, dass sich Software-Projekte a priori nicht exakt vorausplanen lassen. Das zentrale Prinzip sind daher kurzzyklische Intervalle von zwei- bis vierwöchigen „Sprints“, die die Entwicklungszeit unterteilen. Am Ende jedes Sprints muss von jedem Team bereits lauffähige Software („Usable Software“) vorgelegt werden, die dann schrittweise von Sprint zu Sprint erweitert, integriert und ausgebaut wird. Zu Beginn werden daher mit dem Kunden die zentralen Features der Software bestimmt und in eine Liste von Items (den Backlog) überführt, die dann über den Projektverlauf kontinuierlich aktualisiert wird. Die einzelnen Items werden erst von Sprint zu Sprint detailliert beschrieben und umgesetzt. Das kurzzyklische Vorgehen wird schließlich auch auf die Meeting-Routinen der Teams übertragen: Diese sollen sich z. B. täglich zum sog. „Daily Scrum" treffen, in dem sich alle Team-Mitglieder gegenseitig über den jeweiligen Arbeitsfortschritt in Kenntnis setzen.

Mit Scrum entstehen auch neue Rollen und eine veränderte Aufgabenteilung im Projekt. Insbesondere die Rolle des klassischen Projektleiters fällt weg. Stattdessen gibt es den sog. Product Owner, der gegenüber dem Team die Perspektive des Kunden vertritt. Anders als ein Projektleiter kann er jedoch nicht formell die Arbeitsteilung sowie die Zeit- und Kapazitätsplanung des Entwicklerteams bestimmen oder kontrollieren. Diese Aufgaben sollen idealtypisch wiederum eigenverantwortlich vom Team selbst übernommen werden, welches deshalb als empowert bezeichnet wird. Gerade weil dem Team eine tragende Bedeutung zugedacht wird, ist für die (soziale) Integration des Teams eine eigene Rolle, die des sog. Scrum Masters, vorgesehen.

Dabei ist wichtig zu sehen, dass die neuen Anforderungen an Organisation und Mitarbeitende keinesfalls Ausdruck eines kohärenten und abgeschlossenen Konzepts sind. Unsere empirischen Untersuchungen zeigen vielmehr, dass die Vorstellungen davon, was unter „Agilität“ und ,,agile Organisation“ zu verstehen ist, vielfältig und unterschiedlich ausgereift sind. Je nach Organisation und Unternehmensbereich lassen sich zudem unterschiedliche Deutungen und Spielarten identifizieren. Insofern muss man eher von einem strategischen Suchprozess sprechen. Der Umbau zu einer ,,agilen Organisation“ dient damit als Chiffre für die Suche nach neuen Organisationskonzepten.

\section{2 „Mündige Mitarbeitende"}

Mit der agilen Organisation von Arbeit wird zumindest in den Vorreiter-Unternehmen ein neuer Typ von Arbeitskraft adressiert. Gebraucht werden „,mündige Mitarbeitende“, wie es einmal eine Führungskraft in einem unserer Interviews ausgedrückt hat. Damit ist gemeint, dass Unternehmen im Zuge der digitalen Transformation in vielen Bereichen auf Mitarbeitende angewiesen sind, die eng miteinander kollaborieren und dabei eine neue 
„Kultur des Lernens“ entwickeln, die eine hohe Veränderungsbereitschaft mitbringen und selbstorganisiert agieren:

- Das individuelle Expertenwissen soll offengelegt und neue Expertise permanent angeeignet werden: Sowohl über durchgängige IT-basierte Entwicklungs- und Kollaborationsumgebungen als auch durch die Installierung agiler Teams sollen die individuellen „Wissenssilos“ aufgebrochen und die hochqualifizierten Experten in kollaborative und vernetzte Arbeitsprozesse eingebunden werden. Ziel ist es, individuelles Wissen in kollektives bzw. Organisationswissen zu überführen. Damit verbunden sind auch neue Anforderungen im Sinne einer ,kommunikativen Fachlichkeit“" (Bultemeier und Boes 2013).

- Es gilt, veränderungsflexibel zu sein und stets ,über den eigenen Tellerrand hinauszublicken“: Das „Mindset“ der Mitarbeitenden soll an die Bedürfnisse der agilen Organisation angepasst werden. Sie sollen daran gewöhnt werden, dass es keine Gewissheiten mehr gibt und ,nichts mehr fest ist“. Sowohl Standort und Arbeitsplatz als auch Teamzugehörigkeit und Arbeitsinhalt können sich jederzeit ändern. Maßnahmen wie die Regelungen zu mobiler Arbeit oder die Umgestaltung der Bürowelten bis hin zum Verlust fester Arbeitsplätze, aber auch die Anforderung, zeitweilig im Ausland zu arbeiten, zielen darauf, die Flexibilität der Arbeitskräfte zu erhöhen, mit alten Gewohnheiten zu brechen und offen für neue Impulse von außen zu sein.

- Die Mitarbeitenden sollen lernen, eigenverantwortlich zu agieren und nicht mehr lediglich auf Vorgaben und exakte Anweisungen von Vorgesetzten zu reagieren. Umgekehrt müssen Führungskräfte lernen, „loszulassen“ und als „Enabler“ für das Empowerment und die Selbstorganisation des Teams zu fungieren. Ausgehend von der Annahme, dass die hochqualifizierten Experten selbst am besten wissen, wie ihre Arbeit funktioniert, sollen sie diese auch selbst organisieren und planen. Die bestehenden bürokratischen Prozesse sollen von den Mitarbeitenden hinterfragt und in ,intelligente Prozesse“ überführt werden, die immer wieder neu an die jeweils gegebenen Anforderungen angepasst werden können.

Eine wichtige Voraussetzung dafür, dass Mitarbeitende diesen Anforderungen gerecht werden können, ist, dass sie auch über die entsprechenden Freiräume in der Arbeit verfügen - z. B. um sich frei zu vernetzen, um an Informationen zu kommen oder einfach nur, um sich die Zeit zu nehmen, die Dinge in ihrer Tiefe zu durchdenken. Damit birgt diese gegenwärtige Phase der Neueinstellung auf die Herausforderungen des digitalen Umbruchs Chancen, ein Empowerment von Beschäftigten zu fördern. Voraussetzung dafür ist allerdings, dass der Autonomiezuwachs auf Seiten der Beschäftigten in Organisationsstrukturen eingebettet ist, die echte Handlungsfähigkeit sowie nachhaltige Arbeitsbedingungen ermöglichen. Und das ist keineswegs ein Selbstläufer in den Unternehmen. 


\subsection{Empowerment in der agilen Arbeitswelt}

Doch was genau bedeutet Empowerment? Das Konzept des Empowerments wurde in den 70er-Jahren des 20. Jahrhunderts zunächst von der amerikanischen Bürgerrechtsbewegung geprägt und anschließend vor allem von der gemeindebezogenen Sozialen Arbeit aufgegriffen (insbesondere bei Julian Rappaport 1981) (vgl. Simon 1994). Im Mittelpunkt dieses Ansatzes steht eine ressourcenorientierte Perspektive, welche nicht die „Mängel“ von Menschen in den Blick nehmen möchte, sondern vielmehr auf die Stärkung von Potenzialen abzielt. Im Kern geht es dabei um die Unterstützung bei der (Wieder-)Aneignung von Selbstbestimmung über die Umstände des eigenen Lebens. Die meisten Definitionen des Empowerment-Begriffs fokussieren dementsprechend sehr allgemein auf die Förderung der Autonomie und Selbstbestimmtheit von Menschen. Deutlich wird das z. B. bei Rappaport:

„Unter ,empowerment“ verstehe ich, daß es unser Ziel sein sollte, für Menschen die Möglichkeiten zu erweitern, ihr Leben zu bestimmen.“ (Rappaport 1985, S. 269)

Obwohl die Wurzeln des Empowerment-Konzepts im Bereich der Sozialen Arbeit und Gemeindepsychologie liegen, wurde es von der betrieblichen Managementforschung schon früh adaptiert. Bereits in den 70er-Jahren entwickelte Rosabeth Moss Kanter (1977, 1989) das Konzept des strukturellen Empowerments. Es fokussierte darauf, Entscheidungsmacht an niedrigere Hierarchieebenen zu delegieren, um auf diese Art bessere Arbeitsresultate zu erhalten und die Produktivität des Unternehmens zu erhöhen. Kanter benennt fünf Bedingungen in der Arbeit, welche Voraussetzung für das Empowerment der Beschäftigten sind:

1. Möglichkeit, sich selbst weiterzuentwickeln und zu wachsen

2. Zugang zu relevantem Wissen

3. Zugang zu Unterstützungsleistungen (Feedback/Beratung durch Kollegen bzw. Vorgesetzte)

4. Zugang zu adäquaten Ressourcen (Zeit, Mittel)

5. Gelegenheit zum Aufbau und zur Nutzung von persönlichen Netzwerken

Auch wenn Kanter damit wichtige Faktoren von Empowerment hervorhob, galt die isolierte Perspektive auf das strukturelle Empowerment als unvollständig. So merkten Conger und Kanungo (1988, S. 474) an, dass eine alleinige Perspektive auf das strukturelle Empowerment die Selbstwirksamkeit der Mitarbeitenden unberücksichtigt lässt, und Spreitzer (2008) vermisste die Erfassung der Wahrnehmung von bestimmten Strukturen und Praktiken durch die Mitarbeitenden. Sie entwickelte daraufhin das in der Empowerment-Forschung fest etablierte und vielfach validierte Konzept des psychologischen Empowerments (vgl. Arneson und Ekberg 2006; Carless 2004; Laschinger et al. 2001). 
Im Gegensatz zum strukturellen Empowerment steht beim psychologischen Empowerment weniger die tatsächliche Weitergabe von Autorität und Verantwortung im Mittelpunkt, sondern vielmehr die subjektive Wahrnehmung empowernder Arbeitsbedingungen durch die Mitarbeitenden und deren damit verbundene kognitive Zustände (Conger und Kanungo 1988; Spreitzer 1995). Die dahinterliegende Annahme ist, dass das Ausmaß, in dem die Organisation Empowerment-Maßnahmen implementiert, und das Ausmaß, in welchem sich Mitarbeiter empowert fühlen, nicht zwangsläufig übereinstimmen (Spreitzer 2008). Psychologisches Empowerment setzt sich nach Spreitzer aus vier Wahrnehmungen zusammen: das Empfinden von Bedeutsamkeit, Kompetenz, die Erfahrung von Selbstwirksamkeit sowie das Erleben von Einflussnahme (Spreitzer 1995). In ihrer Gesamtheit gelten diese Wahrnehmungen als motivierendes Element für eine proaktive Haltung in der Arbeit (Thomas und Velthouse 1990).

In der Empowerment-Literatur wurde jedoch - nicht zuletzt durch Spreitzer selbst immer wieder darauf verwiesen, dass beide Perspektiven auf Empowerment - also die strukturelle und die psychologische - im Zusammenspiel zu betrachten sind. So betont Spreitzer (1996, 2008), dass eine Interdependenz zwischen der strukturellen und der psychologischen Form von Empowerment besteht und eine Integration beider Perspektiven erforderlich ist, um Empowerment vollständig zu verstehen.

Dieser Auffassung schließen wir uns an. Wir begreifen Empowerment als ein gelingendes Wechselverhältnis zwischen der Bereitschaft der Menschen, sich aktiv einzubringen, und den Rahmenbedingungen, welche die betriebliche Umwelt bereitstellt, um dieses Engagement zu ermöglichen und zu fördern. Empowerment entsteht demnach im Zusammenspiel der Bedingungen, die die Menschen umgeben, und der Wahrnehmung dieser Bedingungen durch die Menschen. Wichtig ist hierbei, dass die Rahmenbedingungen den Beschäftigten echte Handlungsfähigkeit ermöglichen. Das Konzept der Handlungsfähigkeit stammt aus der Kritischen Psychologie. „Handlungsfähigkeit“ wird hier bestimmt als die ,Fähigkeit, im Zusammenschluss mit anderen Verfügung über meine jeweiligen individuell relevanten Lebensbedingungen zu erlangen“ (Holzkamp 1987). Motiviertes Handeln entsteht demnach, wenn die verfolgten Ziele dem Menschen eine Erweiterung der Lebensmöglichkeiten versprechen (ebd.). Empowerment im Arbeitsleben hat nach dieser Lesart also etwas mit dem Verfügen über die objektiven Arbeitsbedingungen zu tun sowie mit der subjektiven Wahrnehmung dieser Bedingungen.

\subsection{Empowerment in der Praxis agiler Teams}

Die Bedeutung, die der Frage des Empowerments in der Praxis einer agilen Arbeitswelt zukommt, konnten wir im Rahmen unserer Forschung zu agilen Teams nachvollziehen. Die empirische Basis dafür bilden zwei Fallstudien in Vorreiterunternehmen, die die Veränderungen der Arbeit im Zuge der Einführung von agilen Methoden in verschiedenen Anwendungsfeldern aufzeigen. Den Kontext bilden dabei die Forschungsprojekte „Lean im Büro - Neue Industrialisierungskonzepte für die Kopfarbeit und die Folgen für Arbeit 
und Beschäftigte“ (gefördert von der Hans-Böckler-Stiftung, 2013-2016) sowie „Empowerment in einer digitalen Arbeitswelt (EdA)“ (gefördert vom BMBF, 2016-2020).

Der Gegenstand der Fallstudie A ist ein großes europäisches Software-Unternehmen, in dem auf der Grundlage agiler Methoden ein neues Produktionsmodell implementiert wurde, das wir in mehreren Erhebungswellen intensiv empirisch beforschen konnten. Dabei konnten wir 70 Interviews mit Beschäftigten und 21 Expertengespräche mit Vertretern des Managements und des Betriebsrats führen. In Fallstudie B geht es um einen Forschungs- und Entwicklungsbereich eines großen, weltweit agierenden Industriekonzerns aus dem Bereich der Metall- und Elektroindustrie, der Scrum als agile Methode der Projektorganisation in einem begrenzten Projektrahmen eingeführt hat. In diese Fallstudie gingen insgesamt zwölf Intensivinterviews mit Beschäftigten und sieben Experteninterviews ein. Fasst man die Ergebnisse der beiden Fallstudien zusammen, so lassen sich verschiedene Entwicklungsstufen von Empowerment bei agilen Teams beobachten:

Werden agile Methoden ausgerollt, bekommen die Teams meist eine Schulung und fangen anschließend an, die neuen Methoden umzusetzen: Die Teams besetzen die neuen agilen Rollen, nutzen ein Scrum- oder Kanban-Board und treffen sich regelmäßig zu Daily Scrums oder Stand-up-Meetings. In dieser Entwicklungsphase handelt es sich um formal agile Teams, welche den Geist der Agilität noch nicht verinnerlicht haben. Die Teams befinden sich vielmehr noch in einer Art Findungsphase, in der viel ausprobiert wird und die neuen Routinen und Rollen noch nicht mit Leben gefüllt sind.

Entscheidend für die weitere Entwicklung der Teams ist nun, ob es gelingt, sie zu empowern. Nur dann kann die Schwelle zu einer wirklich agilen Arbeitskultur überschritten werden, in welcher die Mitarbeitenden zu einem funktionierenden Kollektivteam zusammenwachsen. Statt individueller Wissenssilos dominieren dann kollektive Wissensdomänen und starke Vertrauensbeziehungen. Das Team wird zu einem lernenden Team, es hinterfragt seine bisherigen Routinen und Prozesse und verbessert sich so kontinuierlich. Die Befragten sehen vor allem Sinn in ihrer Arbeit, haben das Gefühl, dass sie „,die Dinge selbst entscheiden und auch zu Ende bringen“ können, und berichten von einer ganz neuen Qualität von Teamarbeit, in der nicht nur ,gemeinsam“, sondern auch wirklich im Sinne eines echten Kollektivteams ,zusammen“ gearbeitet werde. Ein solch konsequentes Empowerment ist in der Praxis allerdings recht selten aufzufinden. Wir konnten es vor allem bei einigen hochqualifizierten Entwicklerteams in sogenannten „Leuchtturmprojekten“ und in kleinen, innovativen Start-up-Unternehmen beobachten.

Wenn es allerdings nicht gelingt, Empowerment zu etablieren und die Schwelle zur agilen Kultur zu überschreiten, dann droht eine Verstetigung zu einem agilen Team ,potemkinschen Typs“. Ohne Empowerment werden die neuen Methoden nur nach außen hin umgesetzt, aber nicht wirklich gelebt. Unterhalb der Oberfläche arbeiten alle weiter wie bisher. Die Menschen unterminieren dann jegliche Formen der Transparenz und der Öffnung von Wissenssilos aus Angst, sich rechtfertigen zu müssen oder sich austauschbar zu machen. Es finden auch keine Lernprozesse statt und das Team stagniert in seiner Entwicklung. 
Dabei zeigen die Ergebnisse deutlich, dass sich gerade in den potemkinschen Teams die Belastungssituation deutlich zuspitzt. Denn ohne Empowerment fehlt ihnen die Möglichkeit, ihre Arbeitslast selbst zu steuern. Dann sind sie der Taktung ihrer Arbeit und dem kurzzyklischen Lieferzwang wehrlos ausgeliefert - was bald zu einer permanenten Überforderung führt. Gleichzeitig fehlt ihnen ohne Empowerment auch das Gefühl von Selbstwirksamkeit, also das Gefühl, etwas bewirken zu können, und die Erfahrung von Sinn in der Arbeit. In unseren Interviews kommt das oft dadurch zum Ausdruck, dass selbst hochqualifizierte Software-Entwickler sich vergleichen mit einem ,einfachen Arbeiter“, der „an einem Fließband“" steht.

Das bedeutet allerdings nicht, dass empowerte Teams vor dauerhaft hohen Beanspruchungen geschützt sind. Entscheidend ist bei ihnen, dass sie als Team lernen, nachhaltig mit den eigenen Ressourcen umzugehen und die steigende Produktivität vor allem für ein gesundes Arbeitstempo und die Stärkung der Sinnperspektive zu nutzen. Ohne ein Selbstverständnis des Teams als ,permanent lernende Organisation“ und ohne das Streben nach kontinuierlicher Verbesserung droht die Gefahr, wieder in alte Muster zurückzufallen und zu stagnieren - bis hin zu einer Abwärtsentwicklung, die schließlich unter die Schwelle der bürokratischen Kultur zurückfällt und in ein ,,verbranntes Team“ münden kann. Notwendig ist es daher, die Lernprozesse im Team nicht lediglich eindimensional auf die Erhöhung der Geschwindigkeit und Verbesserung der Qualität zu richten, sondern vor allem darauf, die Potenziale der Produktivitätssteigerung für eine nachhaltige Geschwindigkeit und die Stärkung salutogener Potenziale, wie z. B. Spaß und Sinnorientierung in der Arbeit, zu nutzen.

Der Blick auf agile Entwicklerteams zeigt also: Empowerment ist der Schlüssel für die erfolgreiche und nachhaltige Gestaltung einer agilen Arbeitskultur. Ohne echtes Empowerment der Teams sind die Beschäftigten dem Belastungspotenzial des neuen Entwicklungsmodells - insbesondere im Zuge der Taktung und des kurzzyklischen Lieferzwangs - wehrlos ausgeliefert, weil ihnen keinerlei Kompensationsstrategien im Team zur Verfügung stehen. Ein ,empowertes Kollektivteam“ verfügt dagegen über die Fähigkeit, seine Arbeitsmenge eigenverantwortlich zu steuern. Mit der gemeinsamen Planung und selbstständigen Schätzung des Arbeitsaufwands entstehen grundsätzlich neue Instrumente, um ein nachhaltiges Arbeitstempo zu ermöglichen. Die Kollaboration im Team und die Entwicklung einer kollektiven Handlungs- und Strategiefähigkeit stellen eine entscheidende Ressource dar, salutogene Potenziale wie die Sinnperspektive oder die Handlungsfähigkeit in der Arbeit zu stärken und Belastungen zu reduzieren.

\subsection{Empowerment stärken: Die wichtigsten Ansatzpunkte}

Die spannende Frage ist jetzt: Was sind die expliziten Einflussfaktoren, die bestimmen, ob Mitarbeitende oder Teams empowert sind? Wir konnten im Rahmen des Verbundprojekts „Empowerment in der digitalen Arbeitswelt - nachhaltige Konzepte für die Digitalisierung 
entwickeln“ (EdA) auf Basis unserer Empirie ${ }^{1}$ in acht verschiedenen Unternehmen aus der IT-, Automobil-, Elektro- sowie Energiebranche die zentralen Ansatzpunkte identifizieren, auf die es ankommt, um ein Empowerment der Mitarbeitenden nachhaltig zu fördern (Abb. 6.1):

Führung: Die Organisation von Führung ist entscheidend bei der Frage, ob es gelingt, das Empowerment der Beschäftigten in den Unternehmen systematisch zu ermöglichen und zu fördern. In agilen Organisationen bedeutet das, dass Führung zu einer gemeinschaftlichen Aufgabe werden muss. Anstelle von hierarchischer Anweisung durch Einzelne wird ein Konzept von Führung benötigt, das auf sozialen Aushandlungsprozessen basiert. Dies betrifft Aushandlungsprozesse im Team ebenso wie zwischen verschiedenen funktionalen Rollen, die jeweils unterschiedliche Perspektiven auf das Ganze darstellen. Eng damit verbunden ist auch die Bereitschaft des Managements, die Autonomie agiler Teams zu akzeptieren. Das Führen empowerter Mitarbeiter bedeutet daher auch, dass sich die Rolle der Führungskraft grundlegend verändert: Statt Kontrolle gewinnen Unterstützung und Beratung als Funktionen von Führung an Bedeutung. Dies beinhaltet beispielsweise die Unterstützung beim Zugang zu wichtigen Ressourcen wie Informationen und

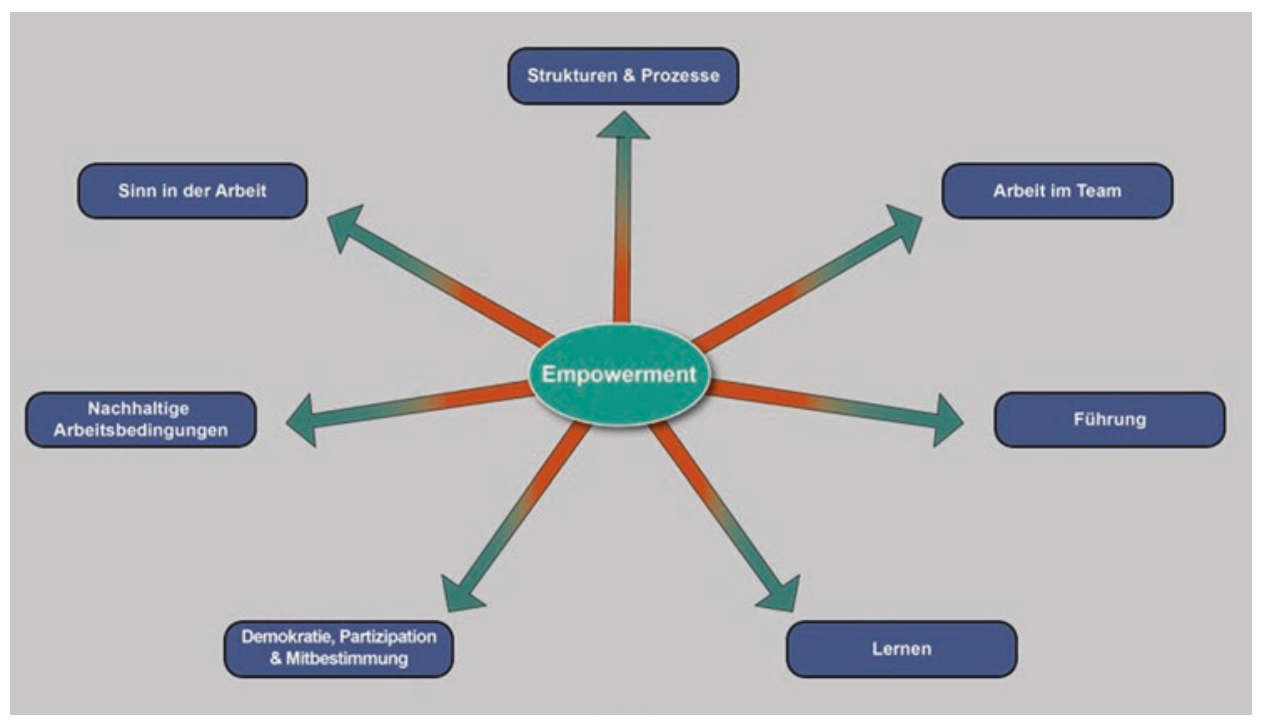

Abb. 6.1 Erfolgsfaktoren für Empowerment, eigene Darstellung

\footnotetext{
${ }^{1}$ Im Rahmen des Verbundprojekts „Empowerment in der digitalen Arbeitswelt - nachhaltige Konzepte für die Digitalisierung entwickeln“ (EdA), gefördert vom Bundesministerium für Bildung und Forschung (BMBF) und vom Europäischen Sozialfonds für Deutschland (ESF), wurden insgesamt 86 Expertengespräche sowie Beschäftigteninterviews in acht verschiedenen Unternehmen aus der IT-, Automobil-, Elektro- sowie Energiebranche geführt. Weitere Informationen: www.eda-projekt.de.
} 
notwendigem Know-how, beim Erwerb von Qualifikationen oder auch bei der Realisierung von Finanz- und Zeitplänen.

Strukturen \& Prozesse: Für das Empowerment der Mitarbeitenden ist es entscheidend, dass sie im Unternehmen Strukturen und Prozesse vorfinden, welche eine flexible Anpassung an sich verändernde Ansprüche und Erfordernisse sowie einen offenen und leichten Austausch über Abteilungsgrenzen hinweg ermöglichen. Hierbei ist wesentlich, inwieweit die Beschäftigten eine Organisationsstruktur erleben, die sie in ihrer Eigeninitiative befördert und nicht durch starre und bürokratische Prozesse behindert. Dazu gehören auch ein offener Umgang mit Informationen, die Möglichkeit, neue Arbeitsformen auszuprobieren, sowie das Angebot von Plattformen und Tools, die eine weitgehend barrierefreie Zusammenarbeit ermöglichen.

Arbeit im Team: Ein empowertes Team verfügt über die entsprechenden Entscheidungsfreiräume, um Arbeitsabläufe selbstbestimmt zu gestalten und eine kollektive Strategie- und Handlungsfähigkeit zu entwickeln. Diese kann es zum einen zur Erschließung von Sinnpotenzialen und persönlicher Entfaltung nutzen und zum anderen zur Grundlage für die Steuerung der eigenen Arbeitsmenge sowie für einen schonenden Umgang mit der eigenen Arbeitskraft machen. Für das Empowerment der Teammitglieder entscheidend ist, ob sich solide und ausgeprägte Vertrauensbeziehungen ausbilden können, die einen konstruktiven Umgang mit Transparenz ermöglichen. Andernfalls können sich Formen eines Gruppen- und Rechtfertigungsdrucks entwickeln, die letztlich auch zur Entstehung neuer Belastungen führen können.

Lernen: Für das Empowerment der Mitarbeitenden ist der Umgang mit dem Thema „Lernen“ im Unternehmen essenziell. Dies geht weit über das Angebot und den Zugang zu Weiterbildungs- und Qualifizierungsmaßnahmen hinaus. Es betrifft sehr viel umfassender die Frage, inwieweit es dem Unternehmen gelingt, durch die Etablierung kontinuierlicher Lernschleifen zu einer ,intelligenten Organisation“ zu werden. Dazu gehört beispielsweise ein konstruktiver Umgang mit Fehlern und Kritik oder auch die Etablierung von Freiräumen für Kreativität und Innovation. Eine wichtige Rolle spielt in diesem Kontext auch die Fähigkeit der Organisation, Erfahrungen und Verbesserungsvorschläge von Mitarbeitenden aufzunehmen und umzusetzen. Und auch das „Lernen voneinander“ ist dabei ein wichtiges Thema: Es geht um die Frage, inwieweit es gelingt, im Unternehmen eine Vertrauenskultur zu erzeugen, die einen offenen Erfahrungs- und Know-how-Transfer zwischen den Mitarbeitenden ermöglicht und aktiv unterstützt.

Demokratie, Partizipation \& Mitbestimmung: In welchem Maße partizipieren Beschäftigte an Unternehmensentscheidungen? Welche Möglichkeiten haben sie, selbst strategische Themen zu setzen und voranzutreiben? In welchem Umfang gibt es im Unternehmen kollektive Vereinbarungen, die das Empowerment der Beschäftigten nachhaltig sichern und das Vertrauen in neue Arbeitsformen stärken? Ein wichtiger Erfolgsfaktor für ein Empowerment der Beschäftigten ist eine beteiligungsorientierte Unternehmenskultur, die Selbstbestimmung durch Mitbestimmung ermöglicht. Gerade die institutionelle Absicherung der neuen Beteiligungsmöglichkeiten agiler Teams kann hier eine wichtige Grundlage schaffen. Dadurch ließe sich verhindern, dass z. B. die Dimensionen des 
Empowerments immer wieder zur Disposition gestellt werden und von den Teams neu verhandelt werden müssen. Stattdessen könnten die Dimensionen des Empowerments über Vereinbarungen zwischen den Sozialparteien verstetigt und den Beschäftigten ein verbriefter Anspruch auf Empowerment gewährt werden.

Nachhaltige Arbeitsbedingungen: Die Möglichkeiten für zeit- und ortsflexible Arbeitsformen wie mobiles Arbeiten oder Home-Office haben mit der Digitalisierung zugenommen. Sie können Beschäftigte wie Führungskräfte prinzipiell in die Lage versetzen, eine bessere Vereinbarkeit von Arbeits- und Privatleben zu erreichen. In der Praxis entscheidet allerdings das Empowerment der Beschäftigten bzw. das Ausmaß ihrer Zeitsouveränität darüber, ob die Flexibilisierung von Arbeitszeit und Arbeitsort der Realisierung nachhaltiger Arbeitsbedingungen dient oder zu einer Verlängerung der Arbeitszeiten sowie ausufernden Verfügbarkeitserwartungen führt. Entscheidend ist hierbei auch die Frage, inwieweit Beschäftigte die Anforderungen der Arbeit mit den sich wandelnden Bedürfnissen in unterschiedlichen Lebensphasen vereinbaren können.

Sinn in der Arbeit: Eine zentrale Komponente des psychologischen Empowerments ist das Empfinden von Bedeutsamkeit in der Arbeit. Hierbei ist es entscheidend, in welchem Umfang es eine Übereinstimmung zwischen den Zielen in der Arbeit und den persönlichen Einstellungen und Wertvorstellungen gibt und ob die Beschäftigten Arbeitsbedingungen vorfinden, in denen sie ihren eigenen Ansprüchen an Inhalt und Qualität in der Arbeit gerecht werden können. Auch die Frage, ob Beschäftigte hierbei das Gefühl der „Handhabbarkeit“ erfahren, also die Überzeugung, den Anforderungen gerecht werden zu können bzw. im Unternehmen etwas bewegen zu können, spielt bei der Erfahrung von Sinn eine wichtige Rolle.

\subsection{Empowerment als humanistischer Gegenentwurf}

Unter dem Eindruck des digitalen Umbruchs beginnt sich die agile Organisation als neue Leitorientierung in den Unternehmen durchzusetzen. Das eröffnet neue Chancen dafür, der Bedeutung des Menschen in der Digitalisierung gerecht zu werden und ihn in den Mittelpunkt zu stellen. Der Schlüssel dafür, dass das gelingen kann, ist die Frage des Empowerments: Nur wenn es gelingt, den Menschen im Arbeitsprozess zu empowern, kann er seiner neuen Rolle in der agilen Organisation gerecht werden.

Die Bedeutung des Empowerments selbst geht allerdings weit über die Frage des Gelingens der agilen Organisation hinaus. Das Konzept des Empowerments markiert vor allem einen humanistischen Gegenentwurf zum Bedrohungsszenario der Digitalisierung als einer Intensivierung von Arbeit und Belastung an digitalen Fließbändern, als Vernichter von Arbeitsplätzen und einer sicheren Zukunftsperspektive sowie als Beschleuniger von Überwachung und Kontrolle in Arbeit und Gesellschaft. Dagegen steht Empowerment für die Perspektive eines Aufbruchs in eine neue Humanisierung der Arbeitswelt, in der die Möglichkeiten der Digitalisierung für die Menschen genutzt werden - und nicht gegen sie! Und zwar indem der Mensch selbst zum Gestalter des Umbruchs wird. Damit dieser 
Gegenentwurf sein Potenzial sowohl in den Unternehmen als auch in der Gesellschaft voll entfalten kann, kommt es darauf an, die reale Bedeutung des Empowerments in der Praxis auszubauen und zu entwickeln - und zwar: von einem Privileg einer kleinen Minderheit in unserer Arbeitsgesellschaft hin zu einer Art „kategorischem Imperativ“ der digitalen Arbeitswelt und zu einer gesellschaftlichen Leitorientierung für die Gestaltung des digitalen Wandels.

Unsere Überlegungen und empirischen Befunde zeigen, dass mit dem Konzept der Agilität im Zuge der digitalen Transformation eine neue Leitorientierung in den Unternehmen entstanden ist. Diese zielt darauf, die Grenzen der fordistisch-bürokratischen Organisation zu überwinden, um die Produktivität zu steigern und die zunehmende Komplexität der Produktionsprozesse bewältigen zu können. So sollen mit agilen Konzepten die auf Basis des Informationsraums entstandenen Möglichkeiten zur Überwindung abgeschotteter Silostrukturen, bürokratischer Entscheidungskaskaden sowie des Expertenmodus praktisch wirksam gemacht werden - durch eine Neustrukturierung der Arbeits- und Organisationsprozesse im Team und eine Ausdünnung der Hierarchieebenen bzw. Erhöhung der Führungsspannen. Im Zuge dessen zeigen sich neue Möglichkeiten für eine Ausweitung der direkten Partizipation im Kontext des Empowerments von Mitarbeitenden und agilen Teams.

In unseren Fallstudien lassen sich diesbezüglich teils weitreichende Potenziale im Hinblick auf die Verfügungsmacht der Beschäftigten über ihren Arbeitsprozess erkennen. Entsprechende Dimensionen umfassen hier den Arbeitsumfang sowie die Art der Umsetzung der Projekte (also das „Wie“ in der Arbeit) und können teilweise sogar bis hin zur Möglichkeit reichen, den Arbeitsinhalt zu bestimmen. Doch auch wenn es in der Unternehmenspraxis bereits einige erfolgreiche Beispiele für ein Empowerment von Beschäftigten gibt, haben diese Beispiele eher einen „Leuchtturm“-Charakter und prägen nicht das Bild in der Breite. Unsere empirischen Ergebnisse zeigen deutlich: Agilität bedeutet nicht automatisch Empowerment. Es lassen sich unterschiedliche Spielarten identifizieren - bis hin zu agilen Teams ohne wirkliches Empowerment.

Eine gezielte Gestaltung der betrieblichen Wirklichkeit in Richtung Empowerment ist daher erforderlich. Die identifizierten Ansatzpunkte sind die entscheidenden Hebel, um Empowerment im Unternehmen nachhaltig und konsequent zu fördern. Dabei ist das Empowerment der Schlüssel, die Menschen auf allen Ebenen dazu zu befähigen, die neuen Herausforderungen im Zuge der digitalen Transformation zu bewältigen. Darüber hinaus ist es aber auch entscheidend, durch Empowerment die Menschen selbst zu den Gestaltern des Umbruchs zu machen. Denn nur wenn gemeinsam mit den Menschen eine Vorwärtsstrategie entwickelt wird und nicht über ihre Köpfe hinweg gestaltet wird, kann es gelingen, anstelle von Angst und Verunsicherung bei den Menschen „Lust auf Zukunft“ zu erzeugen. 


\section{Literatur}

Arneson, H., \& Ekberg, K. (2006). Measuring empowerment in working life: A review. Work, $26,37-46$.

Baukrowitz, A., \& Boes, A. (1996). Arbeit in der „Informationsgesellschaft“ - Einige grundsätzliche Überlegungen aus einer (fast schon) ungewohnten Perspektive. In R. Schmiede (Hrsg.), Virtuelle Arbeitswelten - Arbeit, Produktion und Subjekt in der ,Informationsgesellschaft“ (S. 129-158). Berlin: edition sigma.

Beedle, M., \& Schwaber, K. (2002). Agile software development with Scrum. Upper Saddle River: Prentice Hall.

BMAS (Bundesministerium für Arbeit und Soziales). (2015). Arbeit weiter denken. Grünbuch Arbeiten 4.0. https://www.bmas.de/SharedDocs/Downloads/DE/PDF-Publikationen-DinA4/gruenbuch-arbeiten-vier-null.pdf?_blob=publicationFile. Zugegriffen am 26.10.2019.

Boes, A. (1996). Formierung und Emanzipation - Zur Dialektik der Arbeit in der „Informationsgesellschaft“. In R. Schmiede (Hrsg.), Virtuelle Arbeitswelten - Arbeit, Produktion und Subjekt in der „Informationsgesellschaft“ (S. 159-178). Berlin: edition sigma.

Boes, A. (2004). Offshoring in der IT-Industrie - Strategien der Internationalisierung und Auslagerung im Bereich Software und IT-Dienstleistungen. In A. Boes \& M. Schwemmle (Hrsg.), Herausforderung Offshoring - Internationalisierung und Auslagerung von IT-Dienstleistungen (S. 9-140). Düsseldorf: edition der Hans-Böckler-Stiftung.

Boes, A., \& Kämpf, T. (2012). Informatisierung als Produktivkraft: Der informatisierte Produktionsmodus als Basis einer neuen Phase des Kapitalismus. In K. Dörre, D. Sauer \& V. Wittke (Hrsg.), Kapitalismustheorie und Arbeit (S. 316-335). Frankfurt a. M./New York: Campus Verlag.

Boes, A., Bultemeier, A., Kämpf, T., \& Lühr, T. (2016). Arbeitswelt der Zukunft - zwischen ,digitalem Fließband“ und neuer Humanisierung. In L. Schröder \& H.-J. Urban (Hrsg.), Gute Arbeit. Digitale Arbeitswelt - Trends und Anforderungen (S. 227-240). Frankfurt a. M.: Bund-Verlag.

Brynjolfsson, E., \& McAfee, A. (2011). Race against the machine. How the digital revolution is accelerating innovation, driving productivity and irreversibly transforming employment and the economy. Lexington: Digital Frontier Press.

Bultemeier, A., \& Boes, A. (2013). Neue Spielregeln in modernen Unternehmen - Chancen und Risiken für Frauen. In A. Boes, A. Bultemeier \& R. Trinczek (Hrsg.), Karrierechancen von Frauen erfolgreich gestalten (S. 95-165). Wiesbaden: Springer Gabler.

Carless, S. A. (2004). Does psychological Empowerment mediate the relationship between psychological climate and job satisfaction? Journal of Business and Psychology, 18(4), 405-425.

Conger, J. A., \& Kanungo, R. N. (1988). The empowerment process: Integrating theory and practice. The Academy of Management Review, 13, 471-482.

DeMarco, T., \& Lister, T. (1987). Peopleware: Productive projects and teams. New York: Dorset House Publishing Co Inc.

Dingsøyr, T., Dybå, T., \& Moe, N. B. (2010). Agile software development. Current research and future directions. Heidelberg: Springer.

Durward, D., Simmert, B., Peters, C., Blohm, I., \& Leimeister, J. M. (2019). How to empower the workforce - Analyzing internal crowd work as a neo-socio-technical system. In Hawaii International Conference on System Sciences (HICSS). Waikoloa.

Frey, C., \& Osborne, M. A. (2013). The future of employment. How susceptible are jobs to computerisation?. www.oxfordmartin.ox.ac.uk/downloads/academic/The_Future_of_Employment.pdf. Zugegriffen am 26.10.2019.

Hirsch-Kreinsen, H. (2014). Wandel von Produktionsarbeit - „Industrie 4.0“. Soziologisches Arbeitspapier Nr. 38/2014. Herausgeber: Prof. Dr. H. Hirsch-Kreinsen, Prof. Dr. J. Weyer. 
Holzkamp, K. (1987). Grundkonzepte der Kritischen Psychologie. In: Edition DiesterwegHochschule, Heft 1. Reprint in: AG Gewerkschaftliche Schulung und Lehrerfortbildung (Hrsg., 1987): Wi(e) die Anpassung. Texte der Kritischen Psychologie zu Schule und Erziehung. Verlag Schulze-Soltau: 13-19.

Kanter, R. M. (1977). Men and women of the corporation. New York: Basic Books.

Kanter, R. M. (1989). When giants learn to dance. New York/London: Simon \& Schuster.

Laschinger, H. K., Finegan, J., \& Shamian, J. (2001). Impact of structural and psychological empowerment on job strain in nursing work settings. Expanding Kanter's model. Journal of Nursing Administration, 1, 260-272.

Rappaport, J. (1981). In praise of paradox. A social policy of empowerment over prevention. American Journal of Community Psychology, 9(1), 1-25.

Rappaport, J. (1985). Ein Plädoyer für die Widersprüchlichkeit: Ein sozialpolitisches Konzept des „empowerment“ anstelle präventiver Ansätze. Verhaltenstherapie und psychosoziale Praxis, 2, 257-278 (Übersetzung von: In praise of paradox: A social policy of empowerment over prevention. American Journal of Community Psychology, 9, 1-25, 1981).

Simon, L. (1994). The empowerment tradition in American social work: A history. New York: Columbia University Press.

Spreitzer, G. M. (1995). Psychological empowerment in the workplace: Dimensions, measurement, and validation. Academy of Management Journal, 38(5), 1442-1465.

Spreitzer, G. M. (1996). Social structural characteristics of psychological empowerment. Academy of Management Journal, 39(2), 483-504.

Spreitzer, G. M. (2008). Taking stock: A review of more than twenty years of research on empowerment at work. Handbook of Organizational Behavior, 1, 54-72.

Sutherland, J., \& Schwaber, K. (2011). The Scrum papers: Nut, bolts, and origins of an agile framework. http://jeffsutherland.com/ScrumPapers.pdf. Zugegriffen am 26.10.2019.

Thomas, K. W., \& Velthouse, B. A. (1990). Cognitive elements of empowerment: An ,interpretive“ model of intrinsic task motivation. The Academy of Management Review, 15(4), 666-681.

Woodward, E., Surdek, S., \& Ganis, M. (2010). A practical guide to distributed Scrum. Munich: IBM Press.

Open Access Dieses Kapitel wird unter der Creative Commons Namensnennung 4.0 International Lizenz (http://creativecommons.org/licenses/by/4.0/deed.de) veröffentlicht, welche die Nutzung, Vervielfältigung, Bearbeitung, Verbreitung und Wiedergabe in jeglichem Medium und Format erlaubt, sofern Sie den/die ursprünglichen Autor(en) und die Quelle ordnungsgemäß nennen, einen Link zur Creative Commons Lizenz beifügen und angeben, ob Änderungen vorgenommen wurden.

Die in diesem Kapitel enthaltenen Bilder und sonstiges Drittmaterial unterliegen ebenfalls der genannten Creative Commons Lizenz, sofern sich aus der Abbildungslegende nichts anderes ergibt. Sofern das betreffende Material nicht unter der genannten Creative Commons Lizenz steht und die betreffende Handlung nicht nach gesetzlichen Vorschriften erlaubt ist, ist für die oben aufgeführten Weiterverwendungen des Materials die Einwilligung des jeweiligen Rechteinhabers einzuholen.

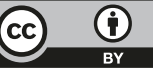

